TAO, Vol. 16, No. 5, 1061-1077, December 2005

\title{
Travel Times and Reflection Points for Reflected P-waves in Transversely Isotropic Media
}

\author{
Young-Fo Chang ${ }^{1, *}$ and Yu-Ting Kuo ${ }^{1}$
}

(Manuscript received 11 March 2004, in final form 1 August 2005)

\begin{abstract}
The travel time curve of reflection waves may not be hyperbolic, and the reflection point may not be located at the midpoint between the source and receiver for $P$-waves reflected from the bottom of a horizontal anisotropic layer. In this study, we used a physical model and numerical calculations to study travel times and reflection points for $P$-waves reflected from transversely isotropic media. We show that the reflection normal moveout (NMO) of the P-waves reflected from the bottom of a horizontal transversely isotropic (TI) layer is hyperbolic within the intermediate offset. The reflection points in the common midpoint (CMP) gather are located at the CMP of the P-waves reflected from TI strata with vertical (VTI) and horizontal (HTI) symmetry axes. However, the reflection points deviate from the CMP for media where the dip angles of the symmetry axes are not vertical and horizontal. The amount of deviation of the reflection points from the CMP depends not only on the offset but also on the dip angle, and the deviations are visualized by physical modeling. Thus, we must be extremely cautious about using the common depth point (CDP) shooting technique and NMO velocity to process seismic data reflected from anisotropic media.
\end{abstract}

(Key words: Normal moveout, Transversely isotropic media, Hyperbolic, Physical modeling)

\section{INTRODUCTION}

The seismic reflection method has been used for oil exploration since the mid-1950s, but a decade later the common depth point (CDP) shooting technique was proposed to improve

\footnotetext{
${ }^{1}$ Institute of Seismology, National Chung Cheng University, Chia-Yi, Taiwan, ROC

* Corresponding author address: Prof. Young-Fo Chang, Institute of Seismology, National Chung Cheng University, Chia-Yi, Taiwan,ROC; E-mail: seichyo@eq.ccu.edu.tw
} 
upon the efficiency and accuracy of that method. Using the CDP shooting technique, we can obtain the velocity of a stratum by analyzing the travel time curve of reflection waves and enhancing the signal to noise ratio $(\mathrm{S} / \mathrm{N})$ by stacking the waves reflected from the common reflection point (CRP) (Dobrin and Savit 1988). However, seismic anisotropy constantly remains in the earth's crust (Crampin 1981; Thomsen 1986; Crampin and Lovell 1991), and the traditional seismic CDP technique is based on an isotropic earth model. In order to further improve the exploration capability of the CDP technique, we must incorporate the processing of seismic data reflected from anisotropic strata.

Thomsen (1986) was the first to derive the normal moveout (NMO) velocity at a shortoffset for waves reflected from transversely isotropic (TI) strata. Among the most notable researchers to attempt to use the nonhyperbolic reflection NMO technique to inverse the anisotropic parameters of a stratum are Alkhalifah and Tsvankin (1995); Grechka and Tsvankin (1998); Al-Dajani and Tsvankin (1998) and Grechka and Tsvankin (2000). In the case of seismic migration which repositions reflected energy from its apparent position to its true subsurface location, care must be taken when treating the reflection points for waves reflected from anisotropic strata (Larner and Cohen 1993; Alkhalifah and Larner 1994; Uzcategui 1995; Alkhalifah 1995; Ball 1995). Uren et al. (1991), Isaac and Lawton (1999) and Vestrum et al. (1999) showed the dramatic shifts in the positioning of reflection points below TI media with a tilted symmetry axis. Vestrum (2002) found that anisotropic depth migration can reduce the depth error on a depth migrated section, so he was able to image sharper reflector terminations and a larger range of reflector angles. The magnitude of the lateral shift of the reflection points from the common middle point (CMP) beneath a tilted transversely isotropic (TTI) overburden depends on the thickness of the overburden, the angle of the tilt, the symmetry-axis velocity as well as the Thomsen anisotropic parameters $\varepsilon$ and $\delta$ (Isaac and Lawton 2004). And if a high quality reflection event below the TTI overburden is observed, then the five parameters can be estimated.

In this study, we used a physical model and numerical calculations to verify whether or not the reflection NMO of the P-waves reflected from TI strata is nonhyperbolic. We are also interested in visualizing the deviations of the reflection points from the CMP.

\section{PHYSICAL MODELING AND NUMERICAL CALCULATIONS}

The vertical transversely isotropic (VTI) medium used in this study was phenolite. Its physical characteristics and direction dependence group velocity is reported in Chang and Chang (2001). Its density is $1.4 \times 10^{3} \mathrm{~kg} \mathrm{~m}^{-2}$, and its elastic constants normalized by density are $A_{11}=15.8, A_{13}=5.0, A_{33}=8.7, A_{44}=2.2$ and $A_{66}=4.5\left(\times 10^{6} \mathrm{~m}^{2} \mathrm{~s}^{-2}\right)$. The velocity-anisotropy $[(\mathrm{Vmax}-\mathrm{Vmin}) / \mathrm{Vmax}]$ of the phenolite for the $\mathrm{P}$ - and $\mathrm{S}$-waves is respectively $25 \%$ and $29 \%$. The three Thomsen non-dimensional anisotropic parameters (Thomsen 1986) of the material are $\delta=0.085, \varepsilon=0.41$ and $\gamma=0.53$. Five cubic, $4 \mathrm{~cm}$ thick blocks were cut from a large piece of VTI phenolite, and the dip angles of the dipping reflectors are $\theta=0^{\circ}$ (VTI), 30 , $45^{\circ}, 60^{\circ}$ and $90^{\circ}$ (HTI, TI medium with a horizontal symmetry axis) (Fig. 1). Also five small cubic $3 \mathrm{~cm}$ thick and $3 \mathrm{~cm}$-wide blocks with individual dip angles of $0^{\circ}, 30^{\circ}, 45^{\circ}, 60^{\circ}$ and $90^{\circ}$ 


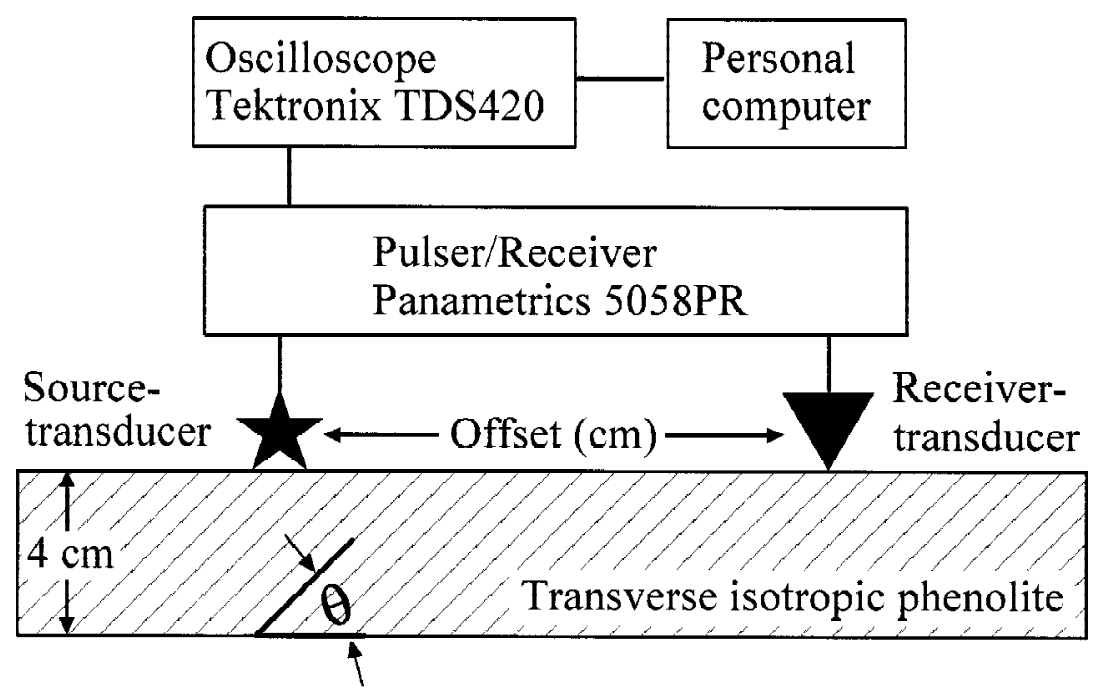

Fig. 1. Apparatus used in our physical modeling system for the double-probe reflection technique. Five TI phenolitic cubic, $4 \mathrm{~cm}$ thick models were made with the dip angles $(\theta)$ of the dipping reflectors being $0^{\circ}, 30^{\circ}, 45^{\circ}$, $60^{\circ}$ and $90^{\circ}$. The black star represents the source, while the black triangle indicates the receiver; they are located on the top surface of the model.

were constructed (Fig. 2). The incidence (sagittal) plane is the symmetry plane of our physical models, and the survey line is in the dip direction (Figs. 1, 2).

In this study, the propagation of ultrasound in the model was simulated as the propagation of seismic waves in the earth. Detailed descriptions of our physical modeling system can be found in Chang and Hsieh (1996) and Chang and Huang (2002). To briefly explain, a pulser/ receiver (Panametrics 5058PR) excites the source-transducer and receives the signal retrieved from the receiver-transducer. Then the signal is sent to an oscilloscope (Tektronix TDS 420) and digitized. A personal computer is used to download the signal from the oscilloscope. A transducer (Panametrics V1091) with a $3 \mathrm{~mm}$ diameter radiates the ultrasound after exciting and/or converting the ultrasound to an electric signal (as shown in Figs. 1,2). The moving of the source and receiver is performed by hand.

Since the direction dependence group velocity, the source and receiver positions and the dip angle of the model were previously known, we used Fermat's principle (i.e., the least travel time technique) to calculate the travel times and reflection points for the P-waves reflected from the bottom of the model. We searched all possible reflection points along the bottom of the model and calculated the travel times of all possible ray paths. Then, it could be assumed that the true ray path was that ray path which had the shortest travel time among all of the possible ray paths. 


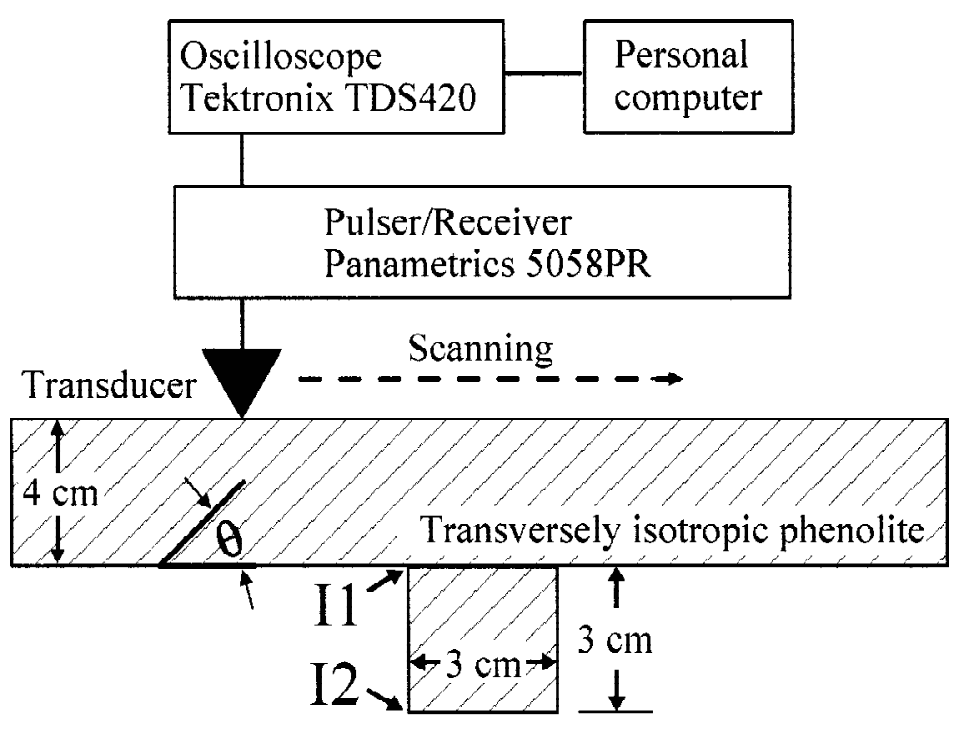

Fig. 2. Apparatus used in our physical modeling system for the single-probe reflection technique. The black triangle is the transducer which serves as both the source and receiver at the same time. Five models were made, and the respective dip angles $(\theta)$ of the dipping reflectors is $0^{\circ}, 30^{\circ}, 45^{\circ}$, $60^{\circ}$ and $90^{\circ}$. Each model was composed of two phenolitic blocks with the same dip angle, and the blocks were held together with honey. I1 is the interface between the two blocks, and $\mathrm{I} 2$ is the air-phenolite interface at the bottom of the model.

Using the double-probe reflection technique, we were able to simulate the acquisition of seismic data in CMP spreading (Fig. 1). The CMP gathers and the ray paths obtained from models where $\theta=0^{\circ}, 30^{\circ}, 45^{\circ}, 60^{\circ}$ and $90^{\circ}$ and are shown in Figs. 3 - 7, respectively. In these figures, we applied the automatic gain control (AGC) to the traces. In Figs. 3a - 7a, the solid lines are the theoretical arrival times of the P-waves which were calculated from Fermat's principle. The dashed lines are the hyperbolic reflection NMOs. The hyperbolic (isotropic) reflection NMO velocity in Figs. 3a - 7a is respectively $3434 \mathrm{~m} \mathrm{~s}^{-1}, 3929 \mathrm{~m} \mathrm{~s}^{-1}, 3583 \mathrm{~m} \mathrm{~s}^{-1}$, $3049 \mathrm{~m} \mathrm{~s}^{-1}$ and $2668 \mathrm{~m} \mathrm{~s}^{-1}$. The P-waves reflected from the bottom of the models can be well predicted using Fermat's principle. They can also be fitted by the hyperbolic curves. The solid lines in Figs. 3b - 7b are the ray paths for the P-waves reflected from the bottom of the models calculated from Fermat's principle. The black stars represent the sources, while the black triangles indicate the receivers. For the models where $\theta=0^{\circ}$ and $90^{\circ}$ (Figs. 3b, 7b), the reflection points are located close to the CMP. For the models where $\theta=30^{\circ}, 45^{\circ}$, and $60^{\circ}$ (Figs. 4b - 6b), they deviate greatly from the CMP.

The zero-offset seismic section can be simulated by the single-probe reflection technique. 


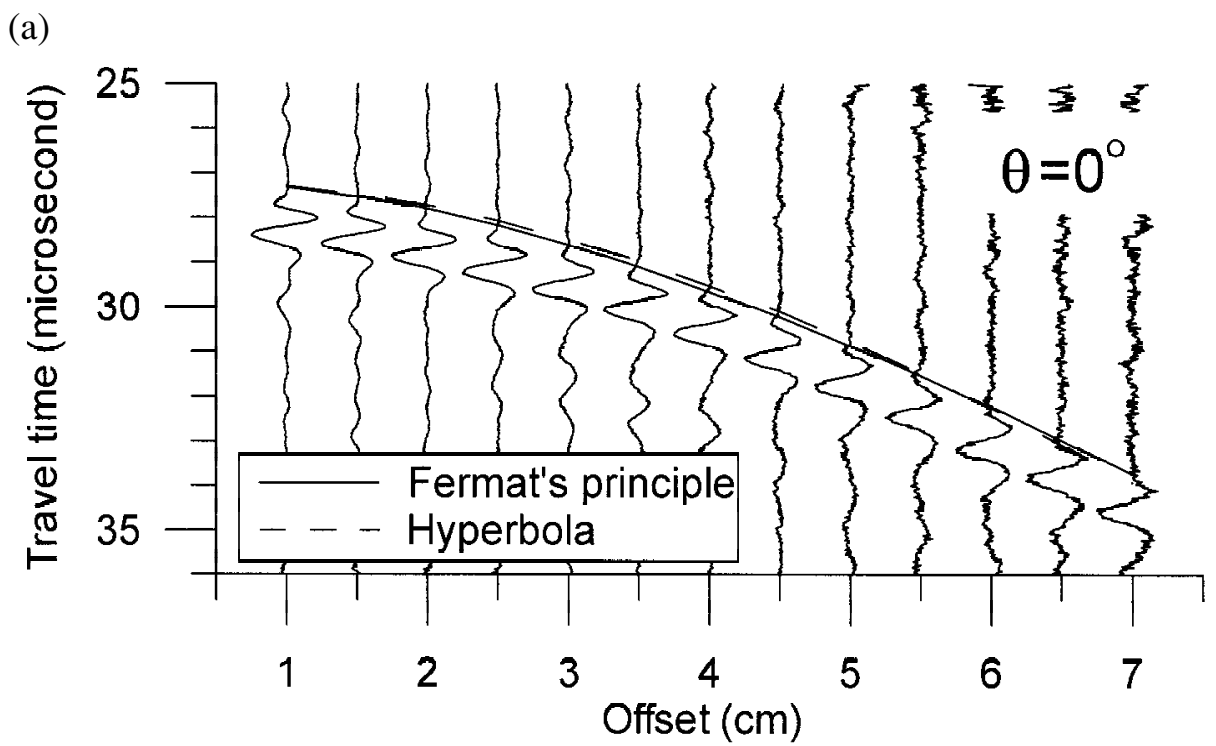

(b)

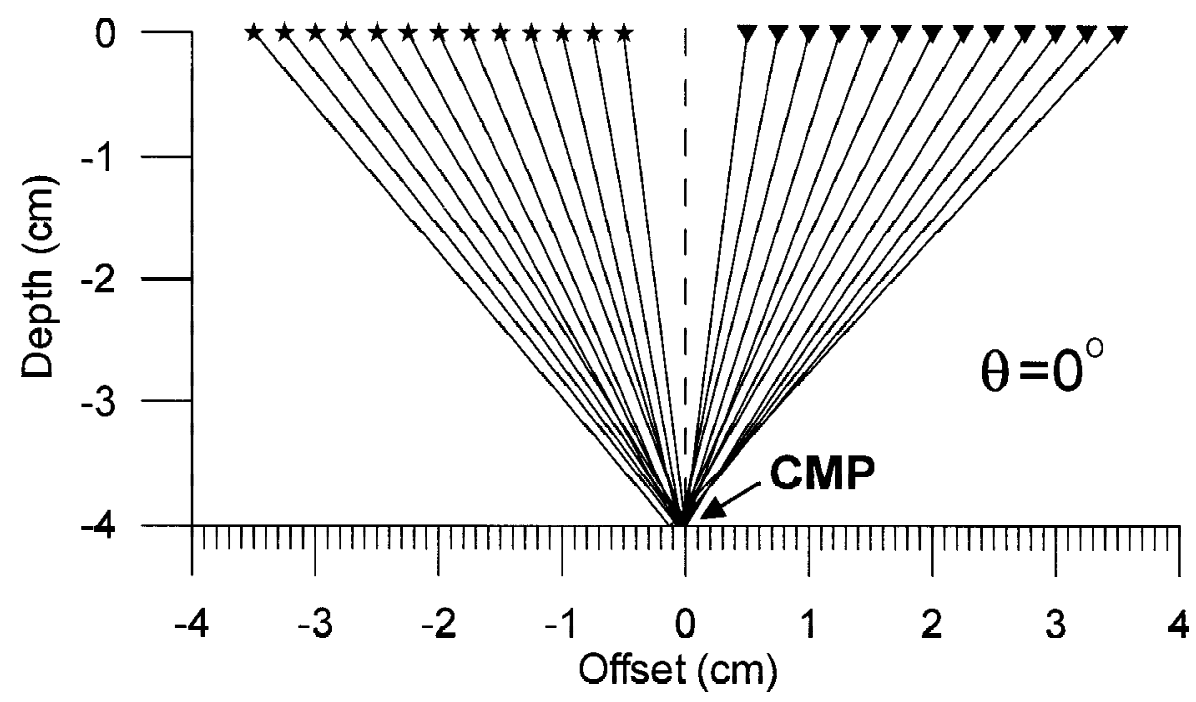

Fig. 3. (a) CMP gather acquired from the model with $\theta=0^{\circ}$. The black stars are the sources, and the black triangles are the receivers. The solid line represents the theoretical arrival times for the P-waves reflected from the bottom of the model. The dashed-line represents the hyperbolic reflection NMO with $3434 \mathrm{~m} \mathrm{~s}^{-1}$ isotropic NMO velocity. Automatic gain control was applied to the traces. (b) Theoretical ray paths for the P-waves reflected from the bottom of the model. 
(a)

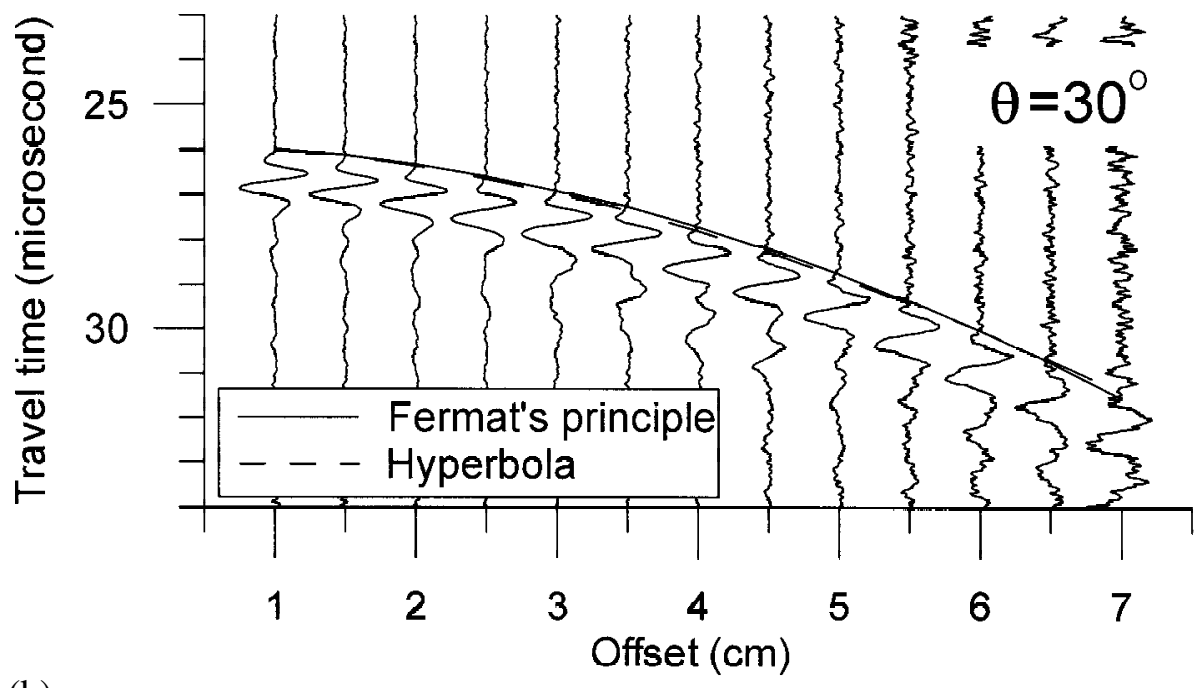

(b)

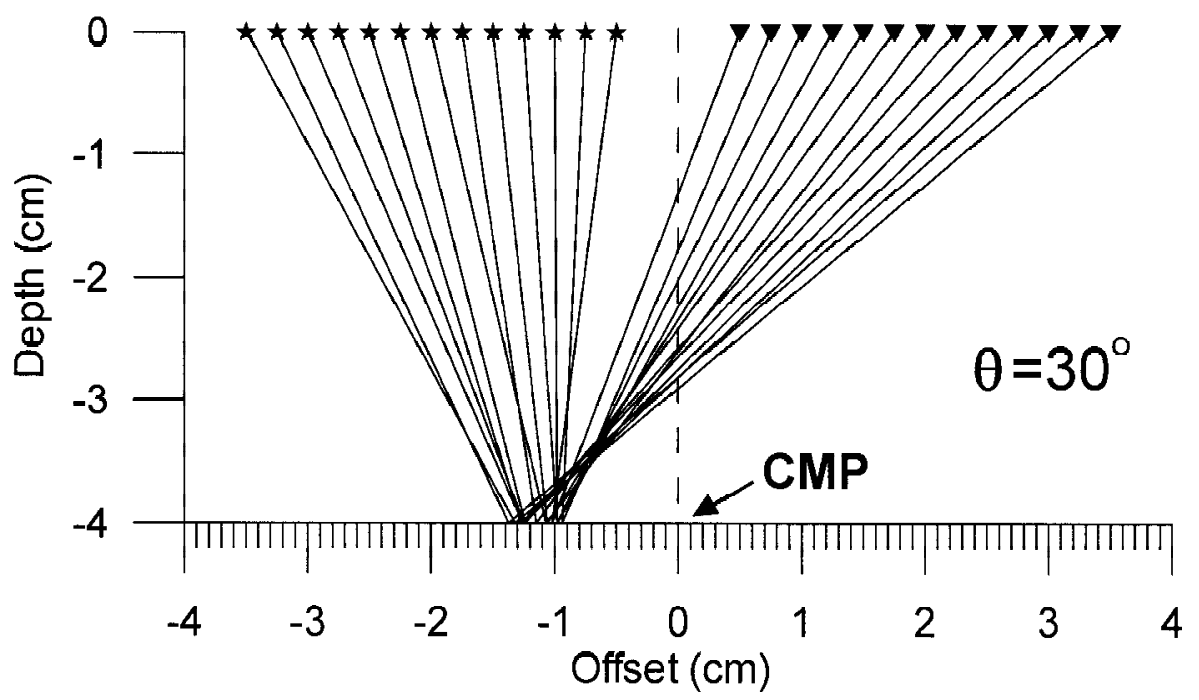

Fig. 4. (a) CMP gather acquired from the model with $\theta=30^{\circ}$. The meaning of the symbols is the same as in Fig. 3. The isotropic NMO velocity is $3929 \mathrm{~m} \mathrm{~s}^{-1}$. (b) Theoretical ray paths for the P-waves reflected from the bottom of the model. 
(a)

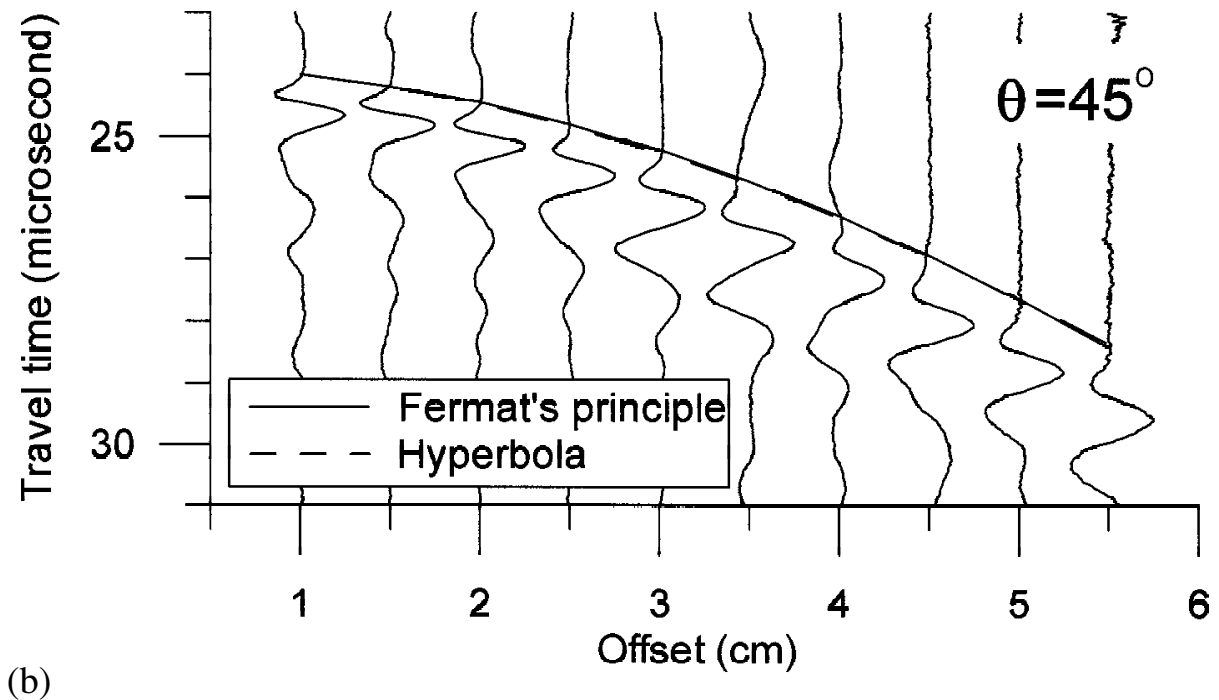

(b)

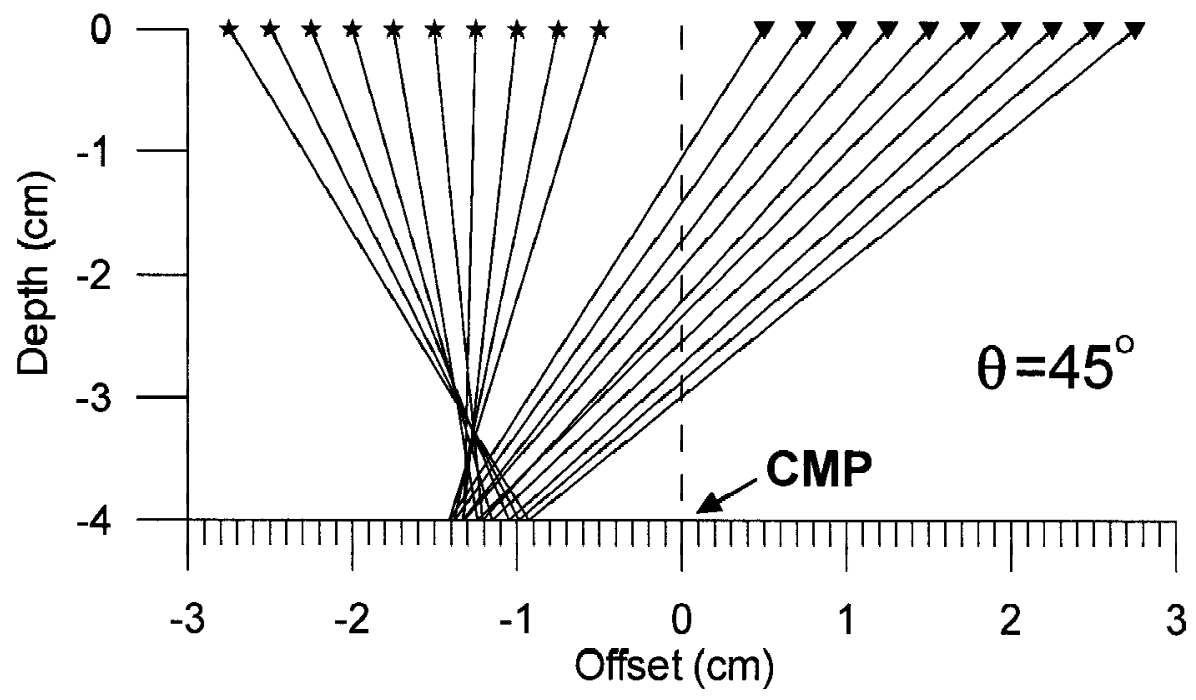

Fig. 5. (a) CMP gather acquired from the model with $\theta=45^{\circ}$. The meaning of the symbols is the same as in Fig. 3. The isotropic NMO velocity is $3583 \mathrm{~m} \mathrm{~s}^{-1}$. (b) Theoretical ray paths for the P-waves reflected from the bottom of the model. 
(a)

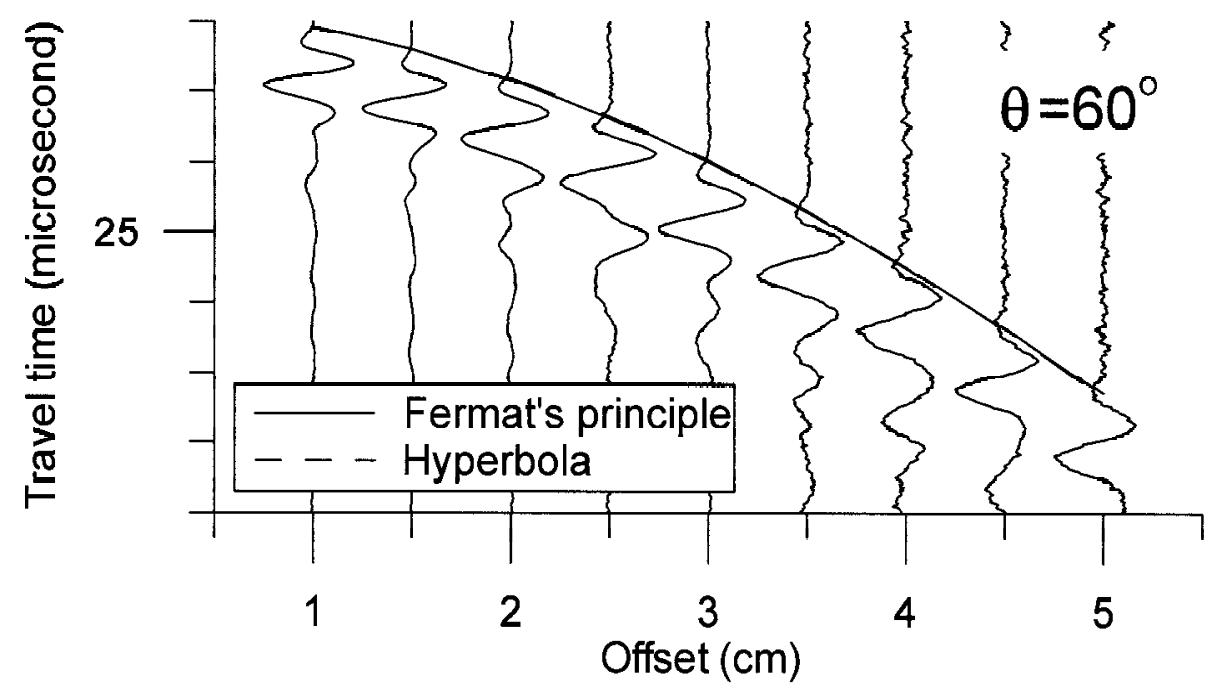

(b)

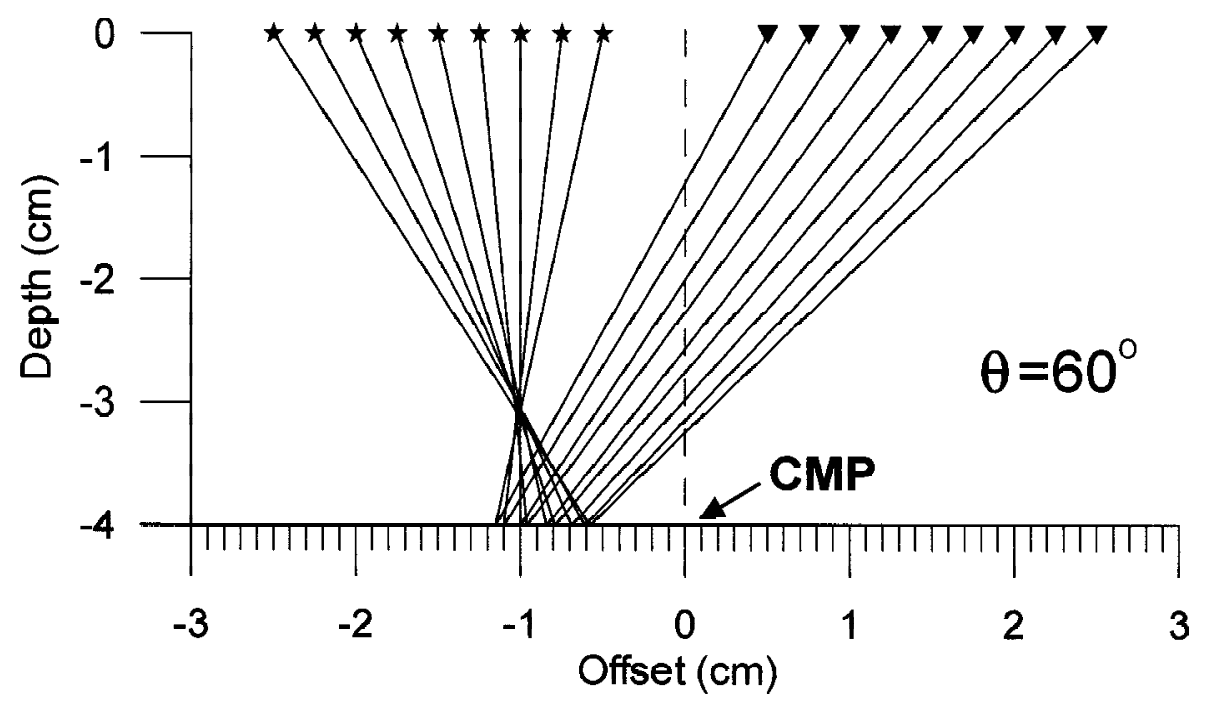

Fig. 6. (a) CMP gather acquired from the model with $\theta=60^{\circ}$. The meaning of the symbols is the same as in Fig. 3. The isotropic NMO velocity is $3049 \mathrm{~m} \mathrm{~s}^{-1}$. (b) Theoretical ray paths for the P-waves reflected from the bottom of the model. 
(a)

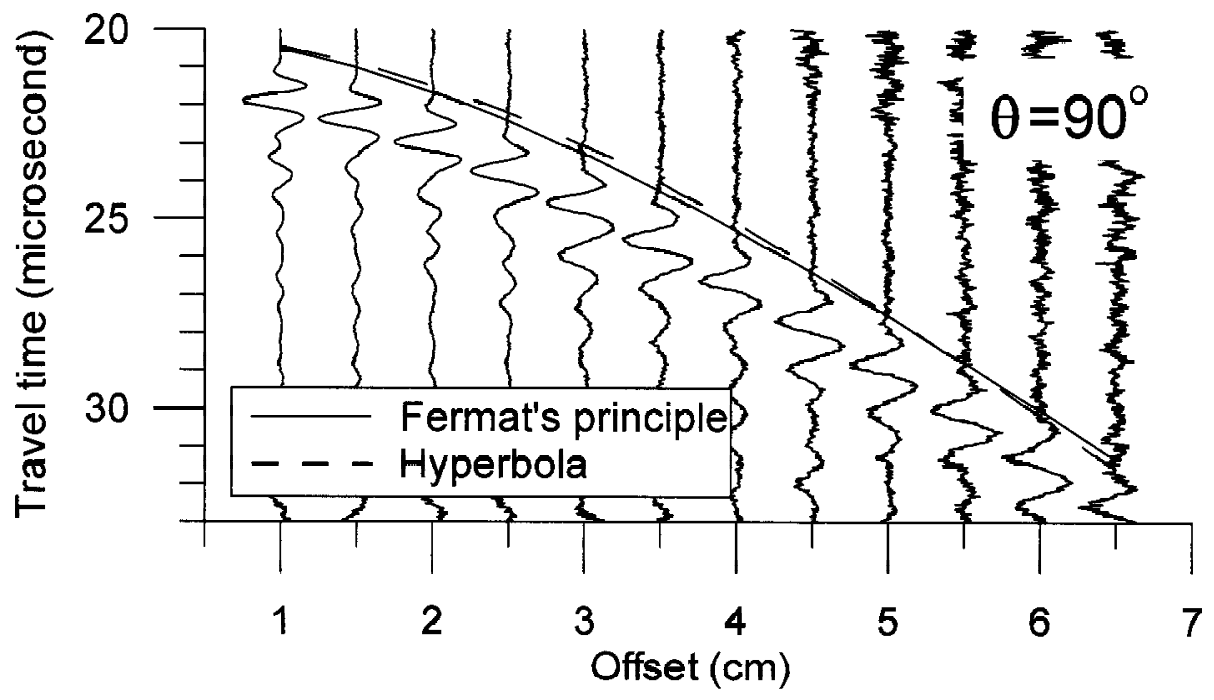

(b)

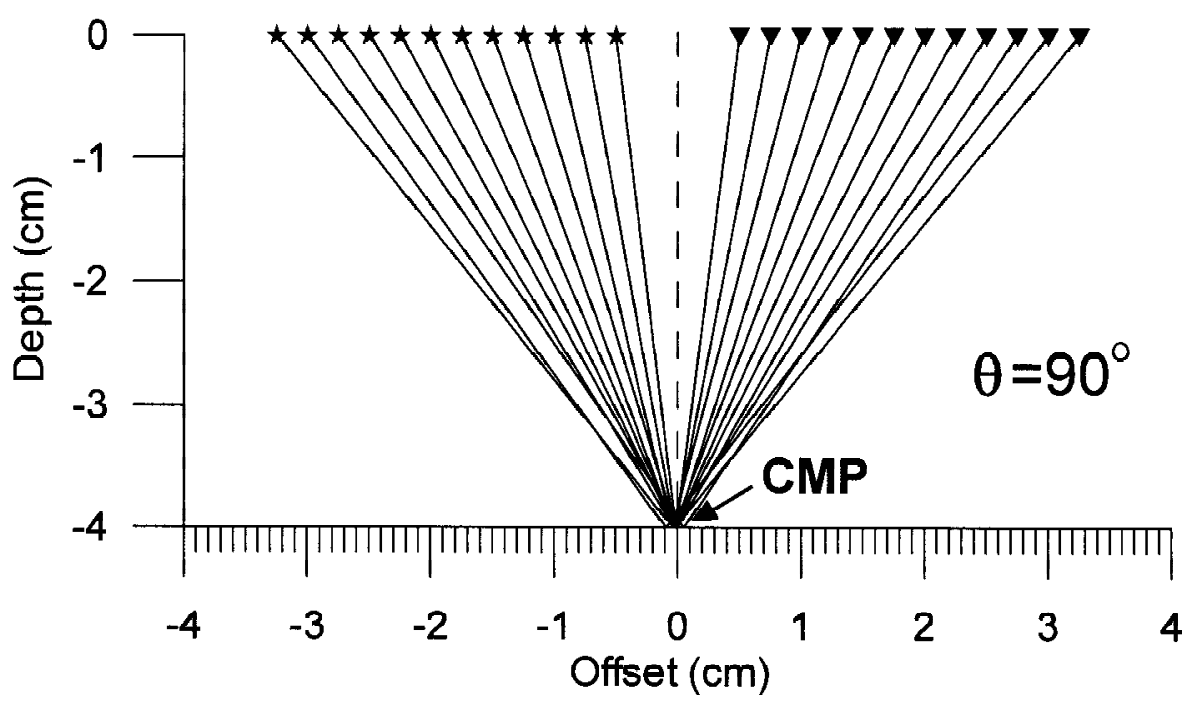

Fig. 7. (a) CMP gather acquired from the model with $\theta=90^{\circ}$. The meaning of the symbols is the same as in Fig. 3. The isotropic NMO velocity is $2668 \mathrm{~m} \mathrm{~s}^{-1}$. (b) Theoretical ray paths for the P-waves reflected from the bottom of the model. 
In this operation mode, the transducer simultaneously serves as both the source and the receiver. The model is composed of two phenolitic blocks with the same dip angle. The thickness of one is $4 \mathrm{~cm}$ and that of the other is $3 \mathrm{~cm}$. They are held together with honey. I1 is the interface between the two blocks, but I2 is the air-phenolite interface at the bottom of the model (Fig. 2). The zero-offset section and the ray paths for the models where $\theta=0^{\circ}, 30^{\circ}, 45^{\circ}, 60^{\circ}$ and $90^{\circ}$ and are shown in Figs. 8 - 12, respectively. In Figs. 8a - 12a, the solid lines denoted by I1 and $\mathrm{I} 2$ are the theoretical arrival times for the P-waves reflected from the I1 and I 2 interfaces, respectively. They are the geometric positions of the interfaces in the sections. The heavy solid lines in Figs. 8b-12b represent the theoretical ray paths for the P-waves reflected from interfaces I1 and I2. It is apparent from these figures that only the traces whose ray paths reach the I 2 interface actually have the energy reflected from that interface. And for these traces, because part of the energy is transmitted into the lower and smaller phenolitic block, the energy reflected from the I1 interface is less than the other traces. For the models where $\theta=0^{\circ}$ and $90^{\circ}$, these traces are directly above the $\mathrm{I} 2$ interface, but they shift to the right about $15 \mathrm{~mm}$ from the interfaces in the models where $\theta=30^{\circ}, 45^{\circ}$, and $60^{\circ}$.

\section{DISCUSSIONS}

The sound beam of ultrasound diverges in the far field. The half-angle is the angle of the beam that spreads between the half- and full-amplitudes. In this study, the half-angle is $7^{\circ}$. The factors controlling the amplitude of the P-waves reflected from the I 2 interface in the zerooffset sections (Figs. 8a-12a) are not only the ray paths but also the beam-spread angle. Therefore, the traces whose ray paths reach the middle of the I 2 interface have greater reflection amplitude than those which simply impinge the edges of the I 2 interface.

Theoretically, the ray paths in Figs. 3b, 7b should be symmetrical about the CMP, but because of numerical errors, they are not. The error in the velocity estimations of our system is $1 \%$, but the error in the travel time calculations is $0.02 \%$. Thus, the numerical error is far less than the experimental error. The numerical travel time error induces a $0.4 \mathrm{~mm}$ deviation of the reflection position in our study, and this deviation is related to the depth of the model. Therefore, the uncertainty in the calculation of the reflection position can be estimated as $0.4 \mathrm{~mm} / 4 \mathrm{~cm}$ $(1 \%)$. For the models where $\theta=30^{\circ}, 45^{\circ}$, and $60^{\circ}$, the shift in the reflection positions from the CMP is about $1.5 \mathrm{~cm}$, and this is far greater than $0.4 \mathrm{~mm}$. Thus, we conclude that the accuracy of the calculation of the reflection position for the P-waves reflected from the bottom of the TI media is acceptable.

The shot gather shows that the reflection NMO of the P-waves reflected from a horizontal anisotropic layer with $\theta=45^{\circ}$ is hyperbolic within the largest offset/depth $=1.4$ (Isaac and Lawton 1999). In our study, the ratio of the largest offset/depth for the models where $\theta=0^{\circ}$, $30^{\circ}, 45^{\circ}, 60^{\circ}$ and $90^{\circ}$ is respectively $1.75,1.75,1.38,1.25$, and 1.63 . Within the intermediate offset and with different dip angles, the reflection NMOs (Figs. 3a - 7a) all remain hyperbolic even though the material has 25\% velocity-anisotropy for the P-waves. However, Alkhalifah and Tsvankin (1995), Grechka and Tsvankin (1998), Al-Dajani and Tsvankin (1998) and Grechka and Tsvankin (2000) have previously used the nonhyperbolic reflection NMO to 


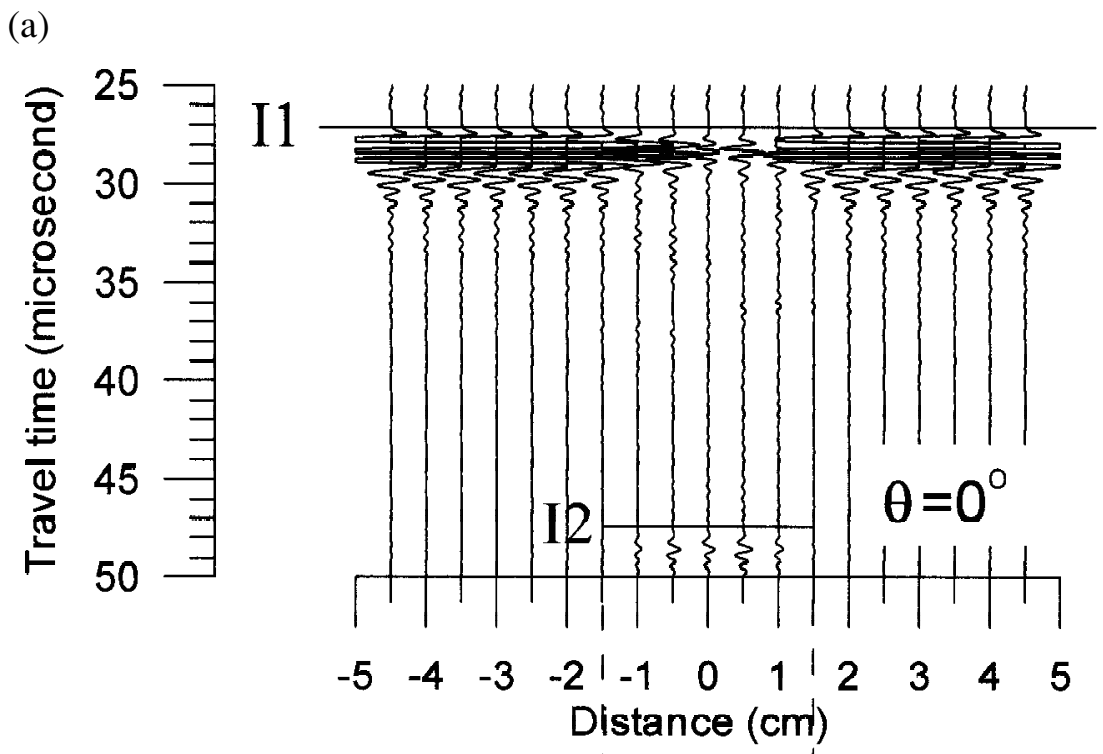

(b)

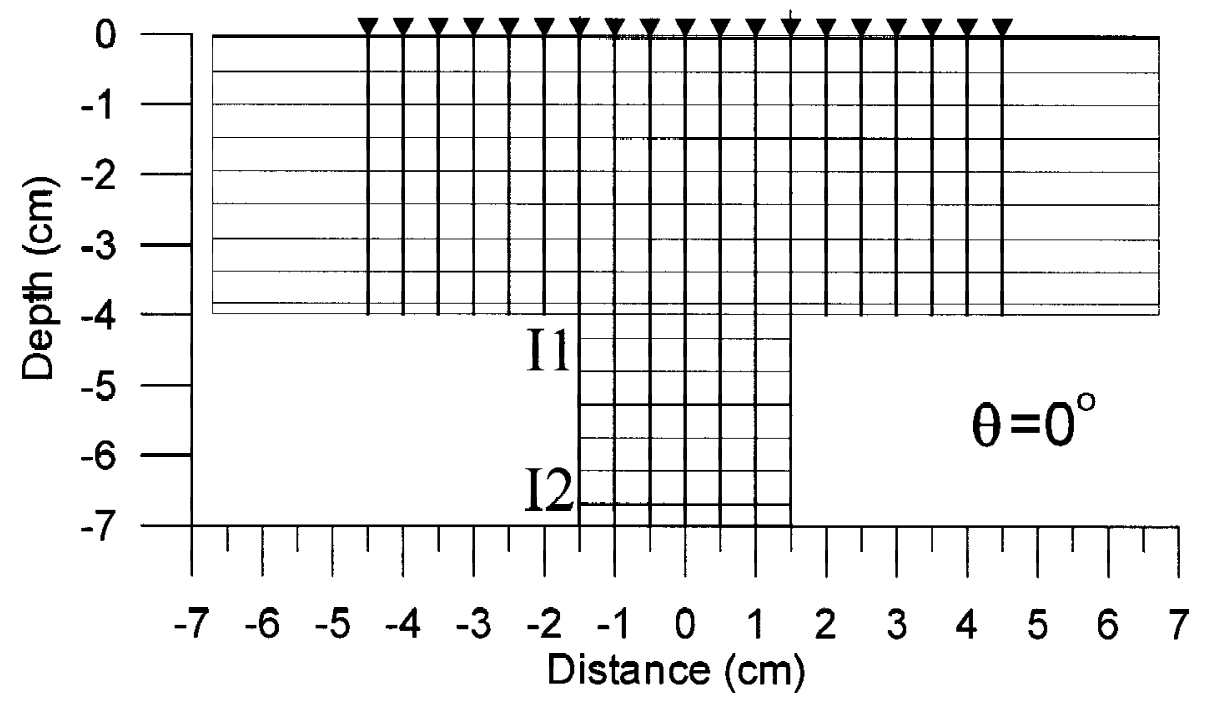

Fig. 8. (a) Zero-offset section acquired from the model with $\theta=0^{\circ}$. The solid lines I1 and I2 are the theoretical arrival times of the P-waves reflected from the I1 and I2 interfaces, respectively. Also, they are the geometric positions of the interfaces in the sections. (b) Heavy solid lines are the ray paths of the P-waves reflected from the I1 and I 2 interfaces. 
(a)

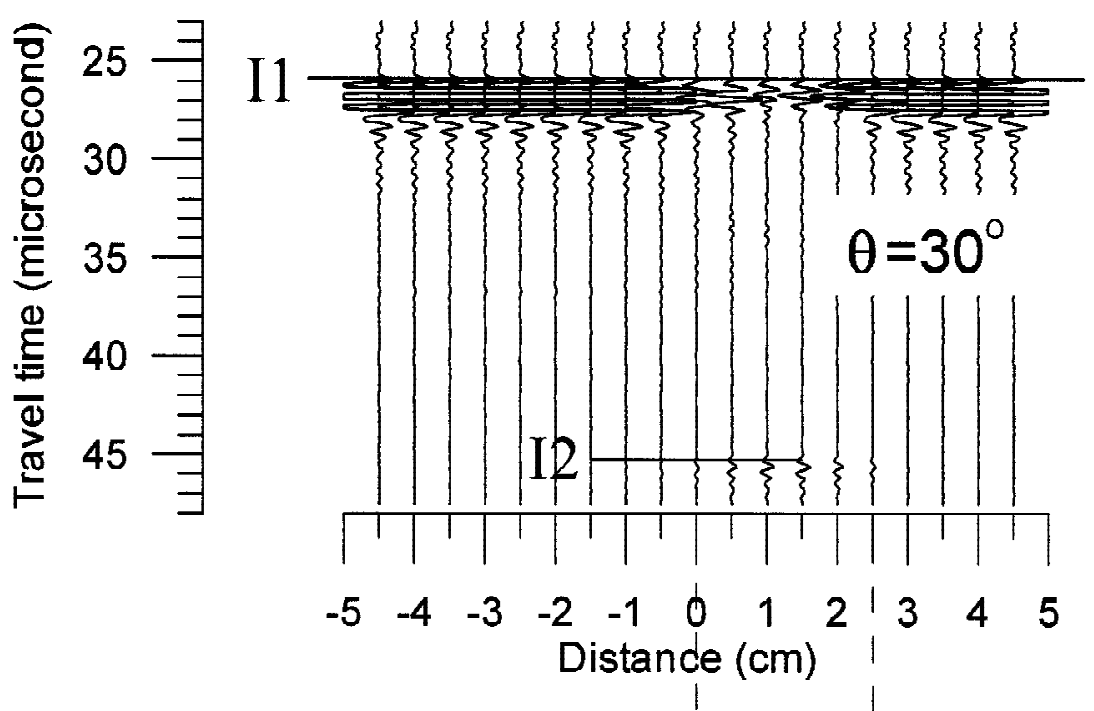

(b)

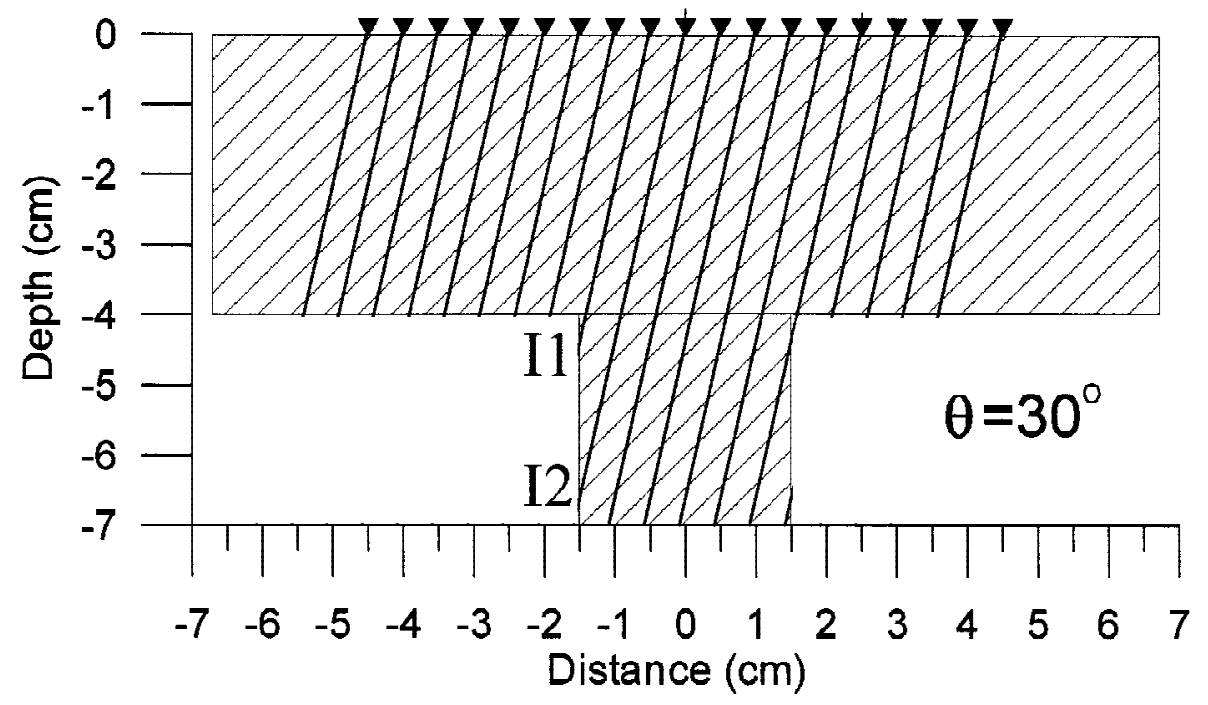

Fig. 9. (a) Zero-offset section acquired from the model with $\theta=30^{\circ}$. (b) Heavy solid lines are the ray paths of the P-waves reflected from the I1 and I2 interfaces. 
(a)

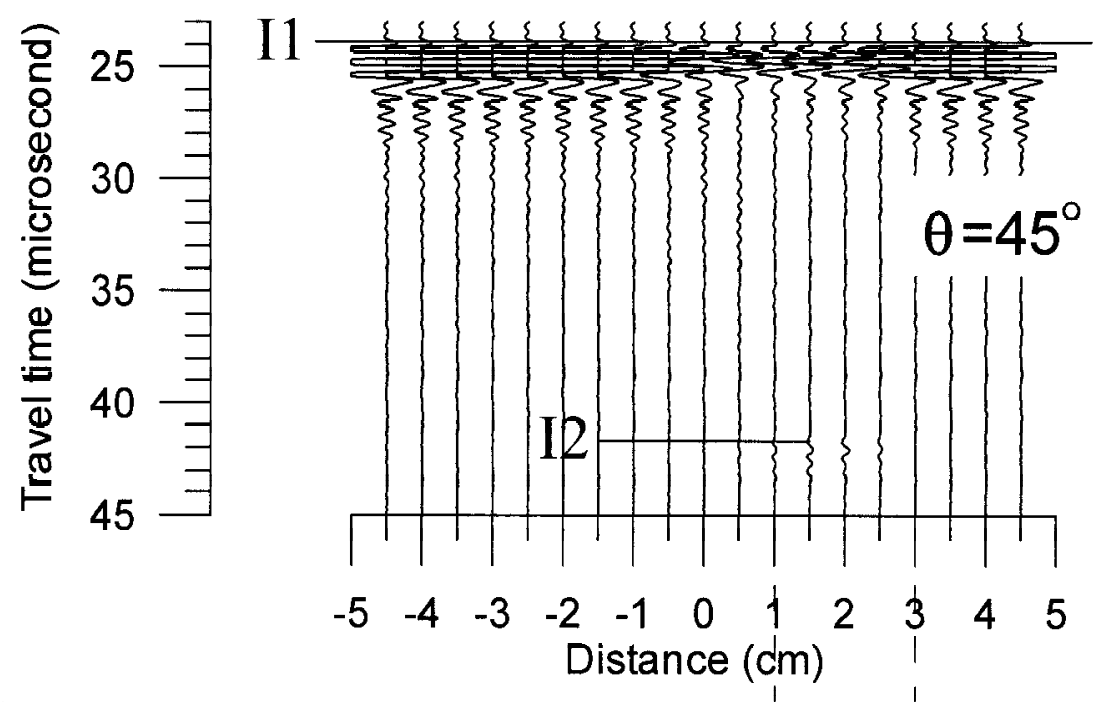

(b)

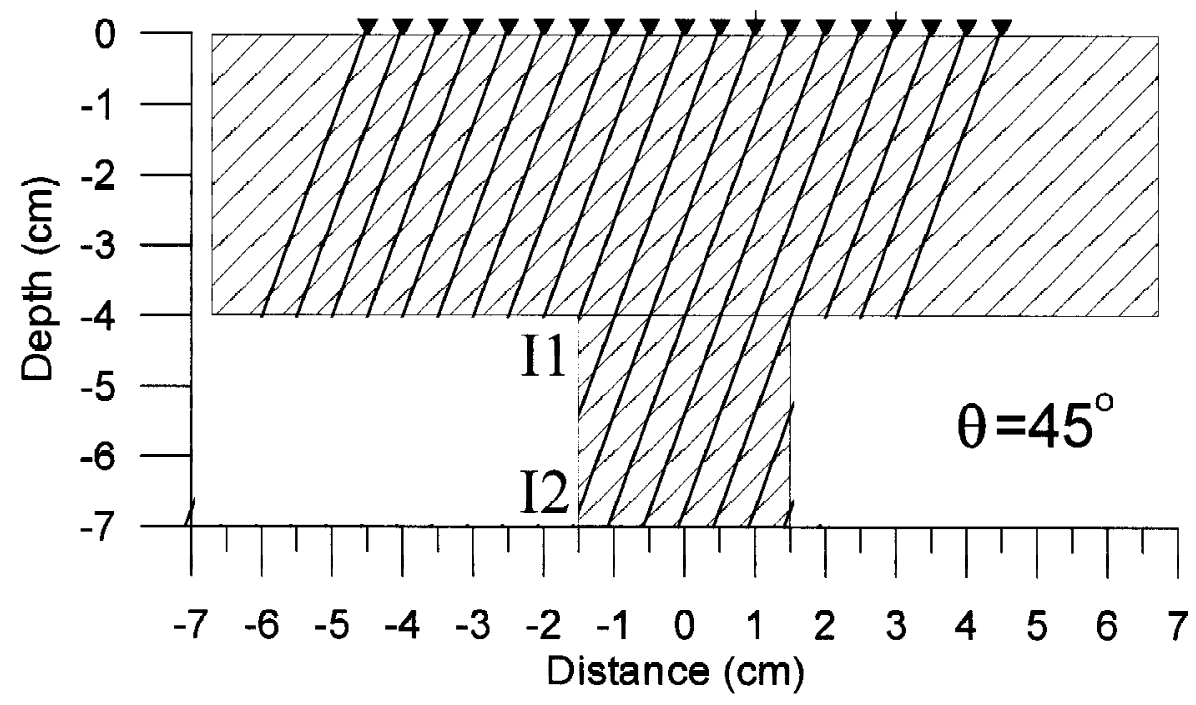

Fig. 10. (a) Zero-offset section acquired from the model with $\theta=45^{\circ}$. (b) Heavy solid lines are the ray paths of the P-waves reflected from the I1 and I2 interfaces. 
(a)

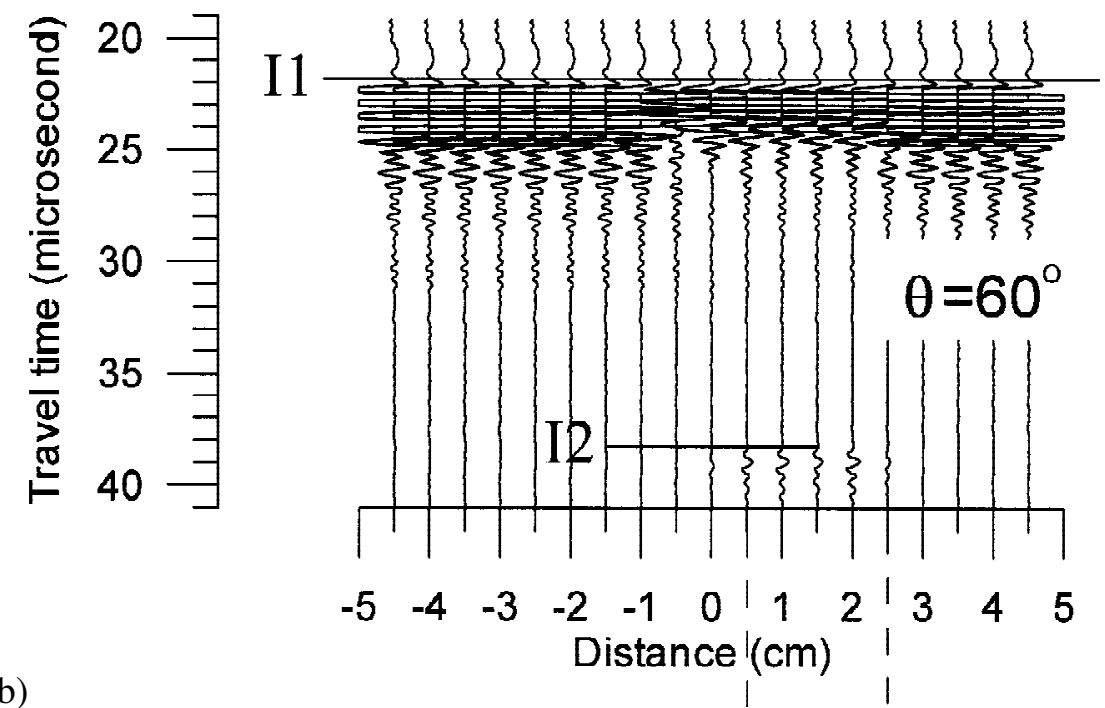

(b)

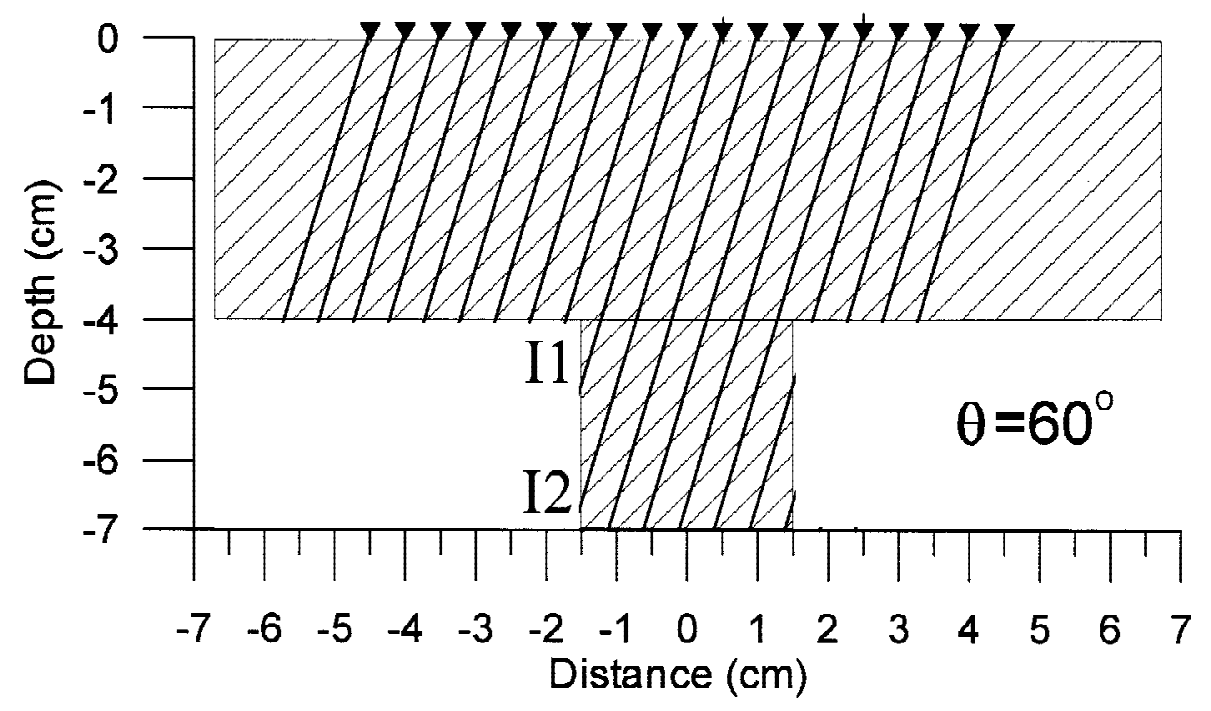

Fig. 11. (a) Zero-offset section acquired from the model with $\theta=60^{\circ}$. (b) Heavy solid lines are the ray paths of the P-waves reflected from the I1 and I2 interfaces. 
(a)

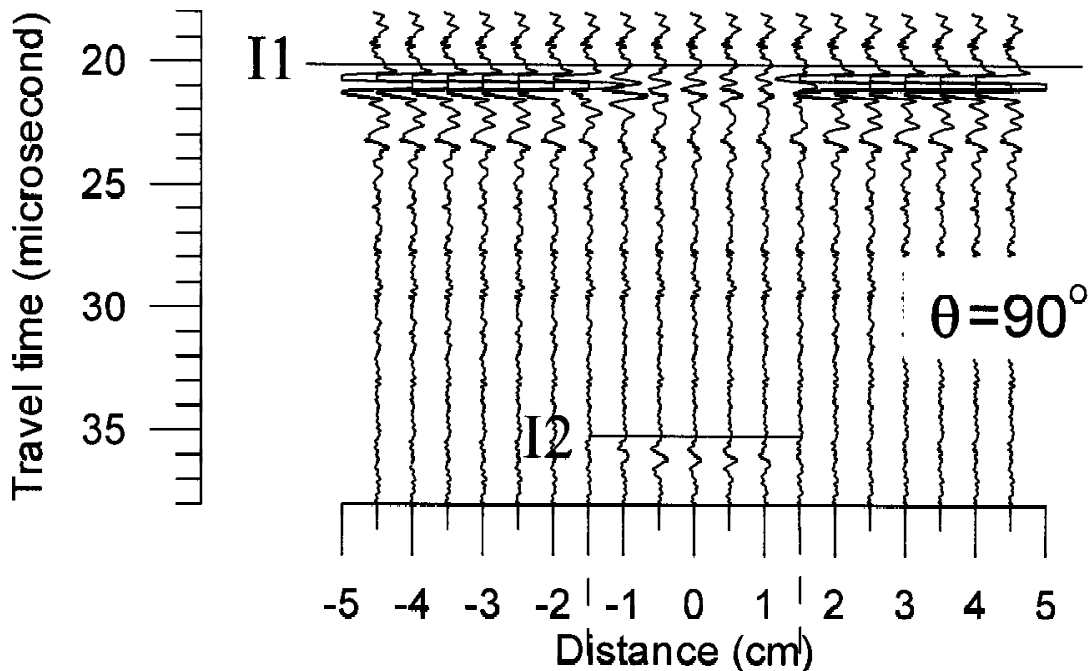

(b)
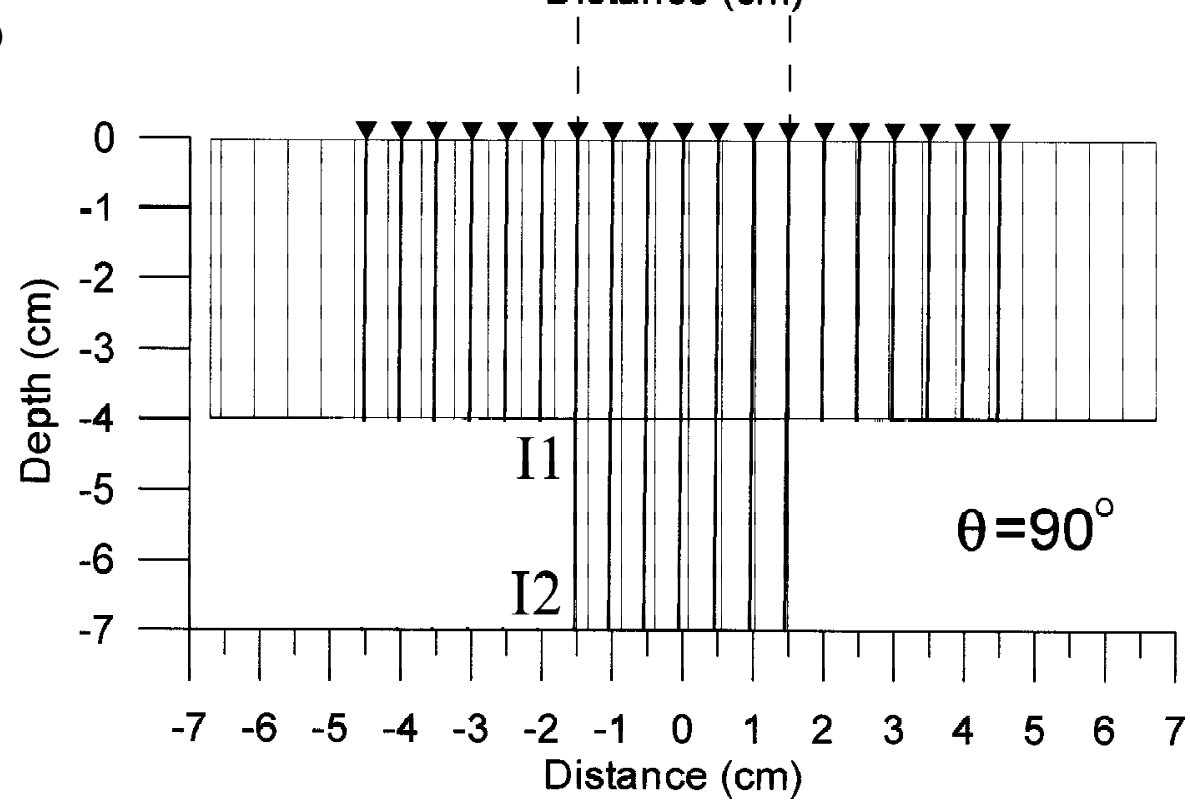

Fig. 12. (a) Zero-offset section acquired from the model with $\theta=90^{\circ}$. (b) Heavy solid lines are the ray paths of the P-waves reflected from the I1 and I2 interfaces. 
derive the anisotropic parameters of a stratum. Intuitively, we used the isotropic NMO velocity to process the seismic data. How did we sense that anisotropy was present from the reflection travel time curve? Our results suggest that the measurable nonhyperbolic reflection NMO can only be detected in highly anisotropic media and at the far offset.

Uren et al. (1991), and Isaac and Lawton (1999) showed that the near offset trace has a greater deviation of the reflection position from the CMP than does the far offset trace. In the present study, this is only the case for the models where $\theta=45^{\circ}$, and $60^{\circ}$. For the model where $\theta=30^{\circ}$, the reflection position of the near offset trace deviates less from the CMP than does the far offset trace. Therefore, the extent of the deviation of the reflection position from the CMP depends on the offset and the dip angle of the tilt.

\section{CONCLUSIONS}

We investigated the travel times and the reflection points for the P-waves reflected from the bottom of the TI media using the physical model along with numerical calculations. The results indicate that:

Using Fermat's principle, we were able to precisely predict the arrival times of P-waves reflected from the bottom of a horizontal TI layer. The reflection NMO of the P-waves within the intermediate offset is hyperbolic. The observable nonhyperbolic reflection NMO can be found only at the far offset and in highly anisotropic media. In the CMP gathers, the reflection points are located at the CMP for only the models where $\theta=0^{\circ}$, and $90^{\circ}$, but for the other dip angle models, the reflection points deviate to the left of the CMP in the down dip direction. For the models where $\theta=45^{\circ}$, and $60^{\circ}$, the near offset trace shows a greater deviation than does the far offset trace, but in the $\theta=30^{\circ}$ model, the near offset trace has a smaller deviation. The deviations of the reflection positions from the CMP can be visualized in the zero-offset sections (Figs. 8 - 12).

By now, the hyperbolic (isotropic) NMO velocity analysis method as well as the CDP shooting technique have widely and successfully been used in seismic exploration for the better of half of a century. Our study shows that we must be especially careful when applying these techniques to process seismic waves reflected from anisotropic strata since the reflection NMO is hyperbolic within the intermediate offset and the reflection point may not be located at the CMP.

Acknowledgments We greatly appreciate the constructive and thoughtful comments of J. Helen Isaac and Chengsung Wang. This research has been financially supported by the National Science Council under grant no. NSC 93-2116-M-194-005.

\section{REFERENCES}

Al-Dajani, A. F., and I. Tsvankin, 1998: Nonhyperbolic reflection moveout for horizontal transverse isotropy. Geophys., 63, 1738-1753. 
Alkhalifah, T., 1995: Gaussian beam depth migration for anisotropic media. Geophys., 60, 1474-1484.

Alkhalifah, T., and I. Tsvankin, 1995: Velocity analysis for transversely isotropic media. Geophys., 60, 1550-1566.

Alkhalifah, T., and K. Larner, 1994: Migration error in transversely isotropic media.Geophys., 59, 1405-1418.

Ball, G., 1995: Estimation of anisotropy and anisotropic 3-D prestack depth migration, offshore Zaire. Geophys., 60, 1495-1513.

Chang, Y. F., and B. S. Huang, 2002: A study of early high-frequency precursors by physical modeling. Terr. Atmos. Ocean. Sci., 13, 1-14.

Chang, Y. F., and C. H. Chang, 2001: Laboratory results for the features of body wave propagation in a transversely isotropic medium. Geophys., 66, 1921-1924.

Chang, Y. F., and C. H. Hsieh, 1996: A note on the characteristics of p coda waves from experimental results. Terr. Atmos. Ocean. Sci., 7, 393-398.

Crampin, S., 1981: A review of wave motion in anisotropic and cracked elastic-media.Wave Motion, 3, 343-391.

Crampin, S., and J. H. Lovell, 1991: A decade of shear-wave splitting in the earth's crust: What does it mean? What use can we make of it? And what should we do next?.Geophys. J. Int., 107, 387-407.

Dobrin, M. B., and C. H. Savit, 1988: Introduction to geophysical prospecting, 4th ed., McGrawHill, New York.

Grechka, V., and I. Tsvankin, 1998: 3-D description of normal moveout in anisotropic inhomogeneous media. Geophys., 63, 1079-1092.

Grechka, V., and I. Tsvankin, 2000: Inversion of azimuthally dependent NMO velocity in transversely isotropic media with a tilted axis of symmetry. Geophys., 65, 232-246.

Isaac, J. H., and D. C. Lawton, 1999: Image mispositioning due to dipping TI media: A physical seismic modeling study. Geophys., 64, 1230-1238.

Isaac, J. H., and D. C. Lawton, 2004: A practical method for estimating effective parameters of anisotropy from reflection seismic data. Geophys., 69, 681-689.

Larner, K. L., and J. K. Cohen, 1993: Migration error in transversely isotropic media with linear velocity variation in depth. Geophys., 58, 1454-1467.

Thomsen, L., 1986: Weak elastic anisotropy. Geophys., 51, 1954-1966.

Uren, N. F., G. H. F. Gardner, and J. A. McDonald, 1991: Anisotropic wave propagation and zero-offset migration. Expl. Geophys., 22, 405-410.

Uzcategui, O., 1995: 2-D depth migration in transversely isotropic media using explicit operators. Geophys., 60, 1819-1829.

Vestrum, R. W., D. C. Lawton, and R. Schmid, 1999: Imaging structures below dipping TI media. Geophys., 64, 1239-1246.

Vestrum, R., 2002: 2D and 3D anisotropic depth migration case histories. SEG Tech. Prog. Expanded Ab., 1256-1259. 